\title{
Correlation analysis between CARMEN variants and alcohol-induced osteonecrosis of the femoral head in the Chinese population
}

Yongchang Guo, Yuju Cao*, Shunguo Gong, Sumei Zhang, Fengzhi Hou, Xinjie Zhang, Jiangeng Hu, Zhimin Yang, Juanjuan Yi, Dan Luo, Xifeng Chen and Jingbo Song

\begin{abstract}
Background: Osteonecrosis of the femoral head (ONFH) is a complicated disease associated with trauma, hormone abuse and excessive alcohol consumption. Polymorphisms of long non-coding RNAs have been also linked with the development of ONFH. Our research aimed to explore the relationship between CARMEN (Cardiac Mesoderm Enhancer-Associated Non-Coding RNA) variants and ONFH risk.

Methods: Our study used Agena MassARRAY Assay to genotype 6 selected single nucleotide polymorphisms (SNPs) in 731 participants (308 alcohol-induced ONFH patients and 423 controls). We used odds ratios (ORs) and 95\% confidence intervals $(\mathrm{Cls})$ to calculate the effect of gene polymorphisms on the occurrence of alcohol-induced ONFH by logistic regression analysis and haplotype analysis.

Results: Our overall analysis illustrated that rs 13177623 and rs12654195 had an association with a reduced risk of ONFH after adjustment for age and gender. We also found that rs13177623, rs12654195 and rs11168100 were associated with a decreased susceptibility to alcohol-induced ONFH in people $\leq 45$ years. In addition, the necrotic sites stratification analysis showed that rs 12654195 was only found to be related to alcohol-induced ONFH risk in the recessive model. In patients with different clinical stages, rs353300 was observed to be associated with a higher incidence of ONFH. Individuals with different genotypes of rs13177623, rs12654195 and rs11168100 had significantly different clinical parameters (cholinesterase, globulin, percentage of neutrophils and the absolute value of lymphocytes).
\end{abstract}

Conclusions: Our data provided new light on the association between CARMEN polymorphisms and alcoholinduced ONFH risk in the Chinese Han population.

Keywords: Osteonecrosis of the femoral head, Chinese, Cardiac mesoderm enhancer-associated non-coding RNA, Polymorphism

\footnotetext{
* Correspondence: caoyuju@126.com

Department of Orthopedics, Zhengzhou Traditional Chinese Hospital of

Orthopaedics, \#1226 East Hanghang Road, Zhengzhou 450000, Henan, China
}

C C The Author(s). 2020 Open Access This article is licensed under a Creative Commons Attribution 4.0 International License, which permits use, sharing, adaptation, distribution and reproduction in any medium or format, as long as you give appropriate credit to the original author(s) and the source, provide a link to the Creative Commons licence, and indicate if changes were made. The images or other third party material in this article are included in the article's Creative Commons licence, unless indicated otherwise in a credit line to the material. If material is not included in the article's Creative Commons licence and your intended use is not permitted by statutory regulation or exceeds the permitted use, you will need to obtain permission directly from the copyright holder. To view a copy of this licence, visit http://creativecommons.org/licenses/by/4.0/ The Creative Commons Public Domain Dedication waiver (http://creativecommons.org/publicdomain/zero/1.0/) applies to the data made available in this article, unless otherwise stated in a credit line to the data. 


\section{Background}

Osteonecrosis of the femoral head (ONFH) is a devastating orthopedic disease, which is characterized by bone cell death due to the damage of microvascular circulation, which is considered to be the result of mechanical vascular disruption, intravascular occlusion and extravascular compression [1, 2]. ONFH usually occurs in people aged $30-50$ years and this refractory disease has a high disability rate [3]. Over the past few decades, the incidence rate of ONFH has been increasing worldwide. Every year, 20,000 people in the United States are diagnosed with ONFH [4], and 2200 people in Japan are diagnosed as ONFH [5]. It is estimated that there are 8.12 million ONFH cases in Chinese people aged 15 years and over [6]. Increasing evidence showed that ONFH is a complex disease associated with many factors, such as trauma [7], genetic factors [8], high-dose corticosteroid use [9] and excessive alcohol consumption. Excessive alcohol intake and steroids are considered to be the main environmental risk factors $[6,10]$. According to statistics in China, 30.7\% of ONFH cases are caused by alcohol [11]. Thereinto, genetic polymorphisms of some genes played critical roles in the occurrence of alcohol-induced ONFH [12-17], such as MMP20, RETN, ApoB, ApoA1, NOS3.

Recently, long non-coding RNAs (lncRNAs), a set of transcribed RNA molecules with a length of more than 200 nucleotides, do not have the ability to encode protein. But these transcripts are able to modulate the target gene expression with the cis-trans regulation. They are involved in the development of many diseases by regulating the mechanisms related to epigenetic modification, transcription and post-processing. As some reports went, not only were they involved in cell proliferation and cell differentiation, but also they were related to tumorigenesis $[18,19]$. In addition, IncRNA, an important regulatory medium, has been reported to be of cardiac lineage specificity in the development process and to have special cellular functions in maintaining cardiac integrity [20, 21].

CARMEN (Cardiac Mesoderm Enhancer-Associated Non-Coding RNA) is also known as CLAP, MIR143HG. Carmen is reported to be highly conserved in mice, and is an important regulatory factor for cardiovascular differentiation. In human, it is found to be active in the heart. And Du et al. found that MIR143HG, as a pathogenic factor, could control the level of RBM24(RNA binding motif protein 24) in Hirschsprung disease (HSCR) positively through the marine spreading of miR143 [22]. Conversely, RBM24 reduced MIR143HG expression by reducing its stability and promoting the synthesis of miR-143. However, the function of MIR143HG in the development of ONFH hasn't been reported until now.
Here, we did a case-control study to investigate the association between CARMEN variants and ONFH risk in the Chinese Han population, which contributes to knowing about the role of CARMEN in the development of $\mathrm{ONFH}$ and is helpful for identifying patients with highrisk alcoholic ONFH.

\section{Methods \\ Subjects}

Totally, 731 male participants (308 alcohol-induced ONFH patients and 423 controls) were recruited by the Zhengzhou Traditional Chinese Hospital of Orthopaedics. Our cases meet the following criteria: 1) The patient's alcohol intake have more than $400 \mathrm{~mL} /$ week [23] (320 g/week, any type of alcoholic beverage) for more than 6 months; 2) ONFH was diagnosed within one year after drinking; 3) Patients had no hyperlipidemia, rheumatoid arthritis, spinal cord cavitation, osteoporosis, decompression sickness, cardiovascular disease and human immunodeficiency virus infection, and no history of steroid use or smoking; 4) The diagnosis of alcoholinduced ONFH was assessed by X-ray, computed tomography $(\mathrm{CT})$, nuclear magnetic resonance imaging (MRI). In the process, ONFH diagnosis was evaluated by Classifcation system [24] originally proposed by Ficat and Arlet. The selection criteria of all healthy people were: 1) Members of the control group had drinking habits and alcohol intake is greater than $400 \mathrm{~mL}$ per week (320 g/ week, any type of alcoholic beverage) for more than 6 months; 2) They had no history of traumatic disease (ONFH, hyperlipidemia, rheumatoid arthritis, spinal cord cavitation, osteoporosis, decompression sickness, cardiovascular disease, steroid use, smoking, etc).

\section{DNA extraction, SNP selection and genotyping}

At least $10 \mathrm{~h}$ after fasting, $5 \mathrm{~mL}$ of venous peripheral blood samples of participants were collected by professional medical personnel and stored in an EDTA anticoagulation tube and $-80^{\circ} \mathrm{C}$ refrigerator. We extracted genomic DNA by using the GoldMag-Mini Whole Blood Genomic DNA Purification Kit (GoldMag Co. Ltd., Xi'an, China). A NanoDrop 2000C spectrophotometer (Thermo Scientifc, Waltham, MA, USA) was applied to detect the DNA concentration and purity. Our present study selected 6 variants located in CARMEN selected from the 1000 Genome Project (https://www.internationalgenome.org/) with minor allele frequencies (MAFs) > $5 \%$ in the global population [25]. Amplification and extension of primers were designed using the Agena MassARRAY Assay Design 3.0 software (Agena, Inc., San Diego, CA, USA). Agena MassARRAY RS1000 (Agena, Inc., San Diego, CA, USA) was used to perform SNP genotyping according to the standard process [16, 25]. 
In the end, we completed the data processing with Agena Bioscience TYPER software, version 4.0 [17].

\section{Statistical analysis}

Age and sex differences between cases and controls were assessed by Student's t-test and Pearson's chi-square, respectively. In addition, we did the genotype distribution of locus in the control group, in order to further explain the good representativeness of the study population. PLINK 1.07 software (Harvard, Boston, MA, USA) [26] was utilized to calculate the association between SNPs and alcohol-induced ONFH risk by logistic regression analysis with ORs and 95\%CI. The version 4.2 of Haploview software (Harvard, Boston, MA, USA) was used to calculate the degree of linkage among these SNPs provided by a linkage disequilibrium (LD) map [27]. $p$-value was two-tailed and $p$-value $<0.05$ was considered statistically significant.

\section{Results}

\section{Basic information of study subjects}

The information of subjects was listed in Table 1. Of population recruited from Department of Orthopedics of Zhengzhou Chinese Hospital, the mean age of 308 cases and 423 controls were $43.47 \pm 11.303$ years and $42.52 \pm 13.135$ years, respectively. No significant difference was found in age and gender between the two groups. In addition, clinical information analysis (hip lesions, clinical stages and course) was also included in the study.

\section{Basic information of selected SNP}

The information of selected SNPs located in CARMEN was shown in Table 2. In Table 2, we listed the chromosome position, specific locations, minor/major alleles, minor allele frequency in cases and controls, HWE (Hardy-Weinberg equilibrium) and allele model. Every

Table 1 The basic information of subjects

\begin{tabular}{llll}
\hline Characteristics & Cases N(\%) & Controls N(\%) & $p$-value \\
\hline Number & 308 & 423 & \\
Age, year (mean \pm SD) & $43.47 \pm 11.303$ & $42.52 \pm 13.135$ & 0.396 \\
$>45$ & $133(43 \%)$ & $198(47 \%)$ & \\
$\leq 45$ & $175(57 \%)$ & $225(53 \%)$ & \\
Hip lesions & & & \\
$\quad$ Unilateral & $66(21 \%)$ & & \\
$\quad$ Bilateral & $242(79 \%)$ & & \\
Clinical stages & & \\
$\quad$ III/IV & $218(71 \%)$ & \\
I/II & $90(29 \%)$ & \\
\hline
\end{tabular}

ONFH Osteonecrosis of the femoral head; TC Total cholesterol; TG Triglycerides; LDL-C Low-density lipoprotein-cholesterol; HDL-C

High-density lipoprotein-cholesterol polymorphism was in accordance with HWE. In the allele model, six variants (rs13177623, rs12654195, rs11168100, rs353303, rs353300 and rs353299) did not appear to be associated with alcohol-induced ONFH.

\section{Relationship between CARMEN variants and alcohol- induced ONFH risk}

Four genetic models (codominant, dominant, recessive and log-additive) were also used to analyze the relationship between six CARMEN variants and alcohol-induced ONFH risk (Table 3). In the codominant model, individuals with rs13177623 G/G genotype had a smaller possibility of ONFH compared to the AA genotype (adjusted $\mathrm{OR}=0.52,95 \% \mathrm{CI}: 0.28-0.94, p=0.031$ ). The recessive model also illustrated that rs13177623 G/G conferred a decreased susceptibility to alcohol-induced ONFH risk in comparison with $\mathrm{A} / \mathrm{A}-\mathrm{A} / \mathrm{G}$ (adjusted $\mathrm{OR}=0.53$, 95\%CI: $0.30-0.95, p=0.033)$. Rs12654195 was also found to be associated with a decreased susceptibility of alcohol-induced ONFH in the codominant (adjusted $\mathrm{OR}=0.53,95 \% \mathrm{CI}: 0.32-0.90, p=0.017)$ and recessive (adjusted $\quad \mathrm{OR}=0.53, \quad 95 \% \mathrm{CI}: \quad 0.32-0.86, \quad p=0.011$ ) models.

\section{Stratification analysis of the association between CARMEN variants and alcohol-induced ONFH risk}

We further assessed the relationship between CARMEN variants and alcohol-induced ONFH risk in $>45$ years groups and $\leq 45$ years groups (Table 4 ). But these sites were only found to be associated with alcohol-induced ONFH in people younger than 45 years. Rs12654195 T was correlated with a reduced risk of alcohol-induced ONFH compared to the allele $G$ (adjusted $\mathrm{OR}=0.69$, 95\%CI: $0.51-0.93, p=0.015)$. There was non-significance between rs13177623 G, rs11168100 $\mathrm{T}$ and alcoholinduced ONFH susceptibility in contrast with wide type allele. In the codominant, recessive and log-additive models, rs13177623 conferred a decreased susceptibility to alcohol-induced ONFH (adjusted $\mathrm{OR}=0.39,95 \% \mathrm{CI}$ : $0.18-0.87, p=0.022$; adjusted $\mathrm{OR}=0.44,95 \% \mathrm{CI}: 0.20$ $0.96, p=0.038$; adjusted $\mathrm{OR}=0.69,95 \% \mathrm{CI}: 0.50-0.95$, $p=0.021)$. Also, rs 12654195 was associated with the risk of alcohol-induced ONFH in four models ( $p=0.008, p=$ $0.033, p=0.019, p=0.008$ ). Whereas, rs11168100 was only related to alcohol-induced ONFH risk in the logadditive model (adjusted $\mathrm{OR}=0.73,95 \% \mathrm{CI}$ : 0.54-0.99, $p=0.045)$.

In addition, we did the necrotic sites stratification analysis to evaluate the association between CARMEN variants and alcohol-induced ONFH risk (bilateral ONFH patients vs controls) shown in Table 5. Rs12654195 was only found to be correlated with alcohol-induced ONFH risk in the recessive model (adjusted OR $=0.60,95 \% \mathrm{CI}$ : $0.35-0.99, p=0.049$ ). 
Table 2 The basic information of selected SNPs located in CARMEN

\begin{tabular}{|c|c|c|c|c|c|c|c|c|c|}
\hline \multirow[t]{2}{*}{ SNP ID } & \multirow[t]{2}{*}{ Gene } & \multirow{2}{*}{$\begin{array}{l}\text { Chromosome } \\
\text { position }\end{array}$} & \multirow[t]{2}{*}{ Role } & \multirow{2}{*}{$\begin{array}{l}\text { Alleles } \\
\text { (minor/major) }\end{array}$} & \multicolumn{2}{|l|}{ MAF } & \multirow{2}{*}{$\begin{array}{l}\text { HWE- } \\
p- \\
\text { value }\end{array}$} & \multirow[t]{2}{*}{ OR $(95 \% \mathrm{Cl})$} & \multirow{2}{*}{$\begin{array}{l}p- \\
\text { value }\end{array}$} \\
\hline & & & & & Case & Control & & & \\
\hline rs13177623 & CARMEN & chr5: 149408144 & Intron & $A / G$ & 0.271 & 0.310 & 0.734 & $0.83(0.66-1.04)$ & 0.110 \\
\hline rs12654195 & CARMEN & chr5: 149409947 & Intron & $\mathrm{G} / \mathrm{T}$ & 0.312 & 0.354 & 0.087 & $0.83(0.66-1.03)$ & 0.090 \\
\hline rs11168100 & CARMEN & chr5: 149413801 & Intron & $\mathrm{A} / \mathrm{T}$ & 0.307 & 0.342 & 0.449 & $0.85(0.68-1.07)$ & 0.162 \\
\hline rs353303 & CARMEN & chr5: 149419554 & Intron & $\mathrm{C} / \mathrm{T}$ & 0.397 & 0.400 & 0.543 & $0.99(0.80-1.22)$ & 0.906 \\
\hline rs353300 & CARMEN & chr5: 149421006 & Intron & $\mathrm{A} / \mathrm{G}$ & 0.516 & 0.480 & 0.206 & $1.16(0.94-1.42)$ & 0.170 \\
\hline rs353299 & CARMEN & chr5: 149421538 & Intron & $\mathrm{A} / \mathrm{G}$ & 0.154 & 0.149 & 0.177 & $1.04(0.78-1.39)$ & 0.781 \\
\hline
\end{tabular}

95\%Cl 95\% Confidence interval; HWE Hardy-Weinberg equilibrium; MAF Minor allele frequency; OR Odds ratio; SNP Single-nucleotide polymorphism $p$-value: Calculated by Pearson $\mathrm{X}^{2}$ test

Analysis of the association between CARMEN variants and alcohol-induced ONFH risk in patients with different clinical stages and clinical parameters

We also investigated the association between CARMEN variants and alcohol-induced ONFH risk in patients with different clinical stages and clinical parameters (Supplementary Table 2 and Supplementary Table 3). In the Supplementary Table 2, stage III and IV individuals were used as the case group, while stage I and II individuals as the control group. The results showed that subjects with rs353300 TC genotype (adjusted OR $=1.83$, 95\%CI: $1.01-3.32, p=0.046$ ) or TT genotype (adjusted $\mathrm{OR}=$ 2.27, 95\%CI: $1.12-4.57, p=0.002$ ) had a higher incidence of ONFH compared with patients with CC genotype, up to 1.83 -fold and 2.27-fold, respectively. When comparing to the $\mathrm{CC}$ genotype in the dominant model, patients with rs353300 T/C-T/T genotype had a higher likelihood of developing into ONFH (adjusted OR $=1.97$, 95\%CI: $1.13-3.44, p=0.017)$. The log-additive model also explained that rs353300 was correlated with an increased risk of alcohol-induced ONFH by 1.52-fold (adjusted OR $=1.52$, 95\%CI: $1.06-2.17, p=0.022$ ).

Moreover, we analyzed the relationship between genotypes of different loci and clinical parameters (cholinesterase, globulin, percentage of neutrophils and absolute value of lymphocytes) in the Supplementary Table 3. We found that the absolute value of lymphocyte (LYMPH) were significantly different among rs13177623 carriers of different genotypes $(p=0.016)$. The content of CHE, GLO and LYMPH were also significantly different among rs12654195 carriers with different genotypes $(p=0.027, p=0.022, p=0.013)$. But, there was no

Table 3 Association between CARMEN variants and ONFH risk

\begin{tabular}{|c|c|c|c|c|c|c|c|c|}
\hline \multirow[t]{2}{*}{ SNP-ID } & \multirow[t]{2}{*}{ Model } & \multirow[t]{2}{*}{ Genotype } & \multicolumn{2}{|c|}{ Frequency } & \multicolumn{2}{|c|}{ Without adjustment } & \multicolumn{2}{|l|}{ With adjustment } \\
\hline & & & Case & Control & OR $(95 \% \mathrm{Cl})$ & $p$-value & OR $(95 \% \mathrm{Cl})$ & $p$-value \\
\hline \multirow[t]{8}{*}{ rs13177623 } & \multirow[t]{3}{*}{ codominant } & $\mathrm{A} / \mathrm{A}$ & 17 & 42 & 1 & & 1 & \\
\hline & & $A / G$ & 133 & 178 & $0.96(0.71-1.30)$ & 0.794 & $0.95(0.70-1.29)$ & 0.726 \\
\hline & & $\mathrm{G} / \mathrm{G}$ & 158 & 203 & $0.52(0.29-0.95)$ & 0.377 & $0.52(0.28-0.94)$ & 0.031 \\
\hline & \multirow[t]{2}{*}{ dominant } & $\mathrm{A} / \mathrm{A}$ & 17 & 42 & 1 & & 1 & \\
\hline & & $\mathrm{A} / \mathrm{G}-\mathrm{G} / \mathrm{G}$ & 291 & 381 & $0.88(0.65-1.18)$ & 0.341 & $0.86(0.64-1.16)$ & 0.332 \\
\hline & \multirow[t]{2}{*}{ recessive } & $\mathrm{A} / \mathrm{A}-\mathrm{A} / \mathrm{G}$ & 150 & 220 & 1 & & 1 & \\
\hline & & $\mathrm{G} / \mathrm{G}$ & 158 & 203 & $0.53(0.30-0.95)$ & 0.033 & $0.53(0.30-0.95)$ & 0.033 \\
\hline & log-additive & - & - & - & $0.82(0.65-1.04)$ & 0.106 & $0.82(0.65-1.03)$ & 0.091 \\
\hline \multirow[t]{8}{*}{ rs12654195 } & \multirow[t]{3}{*}{ codominant } & $\mathrm{G} / \mathrm{G}$ & 25 & 60 & 1 & & 1 & \\
\hline & & $\mathrm{G} / \mathrm{T}$ & 142 & 174 & $1.05(0.77-1.43)$ & 0.771 & $1.04(0.76-1.42)$ & 0.831 \\
\hline & & $\mathrm{T} / \mathrm{T}$ & 141 & 181 & $0.53(0.32-0.90)$ & 0.017 & $0.53(0.32-0.90)$ & 0.017 \\
\hline & \multirow[t]{2}{*}{ dominant } & $\mathrm{G} / \mathrm{G}$ & 25 & 60 & 1 & & 1 & \\
\hline & & $\mathrm{G} / \mathrm{T}-\mathrm{T} / \mathrm{T}$ & 283 & 355 & $0.92(0.68-1.23)$ & 0.563 & $0.91(0.67-1.22)$ & 0.517 \\
\hline & \multirow[t]{2}{*}{ recessive } & $\mathrm{G} / \mathrm{G}-\mathrm{G} / \mathrm{T}$ & 167 & 234 & 1 & & 1 & \\
\hline & & $\mathrm{T} / \mathrm{T}$ & 141 & 181 & $0.52(0.32-0.85)$ & 0.010 & $0.53(0.32-0.86)$ & 0.011 \\
\hline & log-additive & - & - & - & $0.83(0.66-1.03)$ & 0.094 & $0.82(0.66-1.03)$ & 0.087 \\
\hline
\end{tabular}

95\%Cl 95\% Confidence interval; OR Odds ratio; SNP Single-nucleotide polymorphism $p$-value: Calculated by Pearson $x^{2}$ test

Bold type indicates statistical significance $(p<0.05)$ 
Table 4 Association between CARMEN variants and ONFH risk stratified by age

\begin{tabular}{|c|c|c|c|c|c|c|}
\hline \multirow[t]{2}{*}{ SNP } & \multirow[t]{2}{*}{ Model } & \multirow[t]{2}{*}{ Genotype } & \multicolumn{2}{|l|}{$>45 y s$} & \multicolumn{2}{|l|}{$\leq 45 \mathrm{ys}$} \\
\hline & & & OR(95\%Cl) & $p$-value & OR(95\%Cl) & $p$-value \\
\hline \multirow[t]{10}{*}{ rs13177623 } & Allele & A & 1 & & 1 & \\
\hline & & G & $0.97(0.69-1.36)$ & 0.868 & $0.73(0.53-1.00)$ & 0.047 \\
\hline & codominant & $\mathrm{A} / \mathrm{A}$ & 1 & & 1 & \\
\hline & & $A / G$ & $1.15(0.73-1.81)$ & 0.557 & $0.77(0.50-1.19)$ & 0.239 \\
\hline & & $\mathrm{G} / \mathrm{G}$ & $0.71(0.27-1.84)$ & 0.475 & $0.39(0.18-0.87)$ & 0.022 \\
\hline & dominant & $\mathrm{A} / \mathrm{A}$ & 1 & & 1 & \\
\hline & & $\mathrm{A} / \mathrm{G}-\mathrm{G} / \mathrm{G}$ & $1.08(0.7-1.68)$ & 0.726 & $0.69(0.45-1.03)$ & 0.072 \\
\hline & recessive & $\mathrm{A} / \mathrm{A}-\mathrm{A} / \mathrm{G}$ & 1 & & 1 & \\
\hline & & $\mathrm{G} / \mathrm{G}$ & $0.66(0.26-1.66)$ & 0.377 & $0.44(0.20-0.96)$ & 0.038 \\
\hline & log-additive & - & $0.99(0.69-1.41)$ & 0.936 & $0.69(0.50-0.95)$ & 0.021 \\
\hline \multirow[t]{10}{*}{ rs12654195 } & Allele & G & 1 & & 1 & \\
\hline & & $\mathrm{T}$ & $1.03(0.74-1.44)$ & 0.868 & $0.69(0.51-0.93)$ & 0.015 \\
\hline & codominant & $\mathrm{G} / \mathrm{G}$ & 1 & & 1 & \\
\hline & & $\mathrm{G} / \mathrm{T}$ & $1.47(0.92-2.34)$ & 0.108 & $0.74(0.47-1.15)$ & 0.176 \\
\hline & & $\mathrm{T} / \mathrm{T}$ & $0.65(0.27-1.59)$ & 0.349 & $0.41(0.21-0.79)$ & 0.008 \\
\hline & dominant & $\mathrm{G} / \mathrm{G}$ & 1 & & 1 & \\
\hline & & $\mathrm{G} / \mathrm{T}-\mathrm{T} / \mathrm{T}$ & $1.31(0.83-2.05)$ & 0.243 & $0.64(0.42-0.97)$ & 0.033 \\
\hline & recessive & G/G-G/T & 1 & & 1 & \\
\hline & & $\mathrm{T} / \mathrm{T}$ & $0.53(0.23-1.25)$ & 0.147 & $0.47(0.25-0.88)$ & 0.019 \\
\hline & log-additive & - & $1.05(0.74-1.49)$ & 0.796 & $0.67(0.49-0.90)$ & 0.008 \\
\hline \multirow[t]{10}{*}{ rs11168100 } & Allele & A & 1 & & 1 & \\
\hline & & T & $1.00(0.72-1.39)$ & 1.000 & $0.75(0.55-1.01)$ & 0.061 \\
\hline & codominant & $\mathrm{A} / \mathrm{A}$ & 1 & & 1 & \\
\hline & & $A / T$ & $1.39(0.88-2.21)$ & 0.160 & $0.71(0.46-1.11)$ & 0.132 \\
\hline & & $\mathrm{T} / \mathrm{T}$ & $0.63(0.26-1.52)$ & 0.302 & $0.55(0.28-1.08)$ & 0.084 \\
\hline & dominant & $\mathrm{A} / \mathrm{A}$ & 1 & & 1 & \\
\hline & & $\mathrm{A} / \mathrm{T}-\mathrm{T} / \mathrm{T}$ & $1.24(0.8-1.94)$ & 0.340 & $0.67(0.45-1.02)$ & 0.059 \\
\hline & recessive & $\mathrm{A} / \mathrm{A}-\mathrm{A} / \mathrm{T}$ & 1 & & 1 & \\
\hline & & $\mathrm{T} / \mathrm{T}$ & $0.53(0.23-1.23)$ & 0.136 & $0.64(0.34-1.23)$ & 0.181 \\
\hline & log-additive & - & $1.01(0.72-1.43)$ & 0.946 & $0.73(0.54-0.99)$ & 0.045 \\
\hline
\end{tabular}

95\% Cl 95\% Confidence interval; OR Odds ratio; SNP Single-nucleotide polymorphism $p$-value: Calculated by Pearson $x^{2}$ test

Bold type indicates statistical significance $(p<0.05)$

difference in NEUT content among different genotypes of rs12654195 carriers. To our surprise, carriers of different genotypes of rs11168100 had obvious difference in the content of CHE, GLO, NEUT and LYMPH ( $p=$ 0.010, $p=0.011, p=0.048, p=0.014)$.

\section{LD and haplotype analysis}

Among the six variants (rs13177623, rs12654195, rs11168100, rs353303, rs353300 and rs353299), we completed the LD analysis (Fig. 1 and Supplementary Table 4). There was a $1 \mathrm{~kb}$ LD block1 between rs13177623 and rs12654195, and rs11168100, rs353303, rs353300 formed a
$7 \mathrm{~kb}$ LD block2. Totally, AAC haplotype was associated with an increased alcohol-induced ONFH risk by 1.62 -fold (adjusted $\mathrm{OR}=1.62,95 \% \mathrm{CI}: 1.14-2.30, p=0.007$ ). After age stratification analysis, five haplotypes (AG, GT, TA, AA and TC) were related to a decreased alcohol-induced ONFH risk in $\leq 45$ years patients. Haplotype AA showed an increased alcohol-induced ONFH risk in $\leq 45$ years patients (adjusted OR $=1.64,95 \% \mathrm{CI}: 1.20-2.25, p=0.002$ ). And after necrotic sites stratification analysis, AAC haplotype was also found to be associated with an increased alcoholinduced ONFH risk in patients with bilateral necrotic sites (adjusted OR $=1.72,95 \%$ CI: 1.19-2.49, $p=0.004$ ). 
Table 5 Association between CARMEN variants and ONFH risk stratified by necrotic sites

\begin{tabular}{|c|c|c|c|c|c|c|c|c|}
\hline \multirow[t]{2}{*}{ SNP } & \multirow[t]{2}{*}{ Model } & \multirow[t]{2}{*}{ Genotype } & \multicolumn{2}{|c|}{ Frequency } & \multicolumn{2}{|c|}{ Without adjustment } & \multicolumn{2}{|l|}{ With adjustment } \\
\hline & & & Case & Control & $\mathrm{OR}(95 \% \mathrm{Cl})$ & $p$-value & $\mathrm{OR}(95 \% \mathrm{Cl})$ & $p$-value \\
\hline \multirow[t]{8}{*}{ rs12654195 } & codominant & $\mathrm{G} / \mathrm{G}$ & 22 & 60 & 1 & & 1 & \\
\hline & & $\mathrm{G} / \mathrm{T}$ & 111 & 174 & $1.06(0.76-1.48)$ & 0.737 & $1.05(0.75-1.47)$ & 0.780 \\
\hline & & $T / T$ & 109 & 181 & $0.61(0.35-1.05)$ & 0.073 & $0.61(0.35-1.05)$ & 0.074 \\
\hline & dominant & $\mathrm{G} / \mathrm{G}$ & 22 & 60 & 1 & & 1 & \\
\hline & & $\mathrm{G} / \mathrm{T}-\mathrm{T} / \mathrm{T}$ & 220 & 355 & $0.94(0.69-1.30)$ & 0.722 & $0.94(0.68-1.29)$ & 0.688 \\
\hline & recessive & $\mathrm{G} / \mathrm{G}-\mathrm{G} / \mathrm{T}$ & 133 & 234 & 1 & & 1 & \\
\hline & & $\mathrm{T} / \mathrm{T}$ & 109 & 181 & $0.59(0.35-0.99)$ & 0.046 & $0.60(0.35-0.99)$ & 0.049 \\
\hline & log-additive & - & 142 & 174 & $0.86(0.68-1.09)$ & 0.219 & $0.86(0.68-1.09)$ & 0.211 \\
\hline
\end{tabular}

95\% CI 95\% Confidence interval; OR Odds ratio; SNP Single-nucleotide polymorphism $p$-value: Calculated by Pearson $x^{2}$ test

Bold type indicates statistical significance $(p<0.05)$

\section{Discussion}

Our case-control study illustrated that CARMEN variants were related to the risk of ONFH. Rs13177623, rs12654195 and rs11168100 were associated with alcohol-induced ONFH in people younger than 45 years. Rs12654195 was only found to be related to alcoholinduced ONFH risk after the necrotic sites stratification analysis. In patients with different clinical stages, rs353300 was observed to be associated with a higher incidence of ONFH. While, individuals with different genotypes of rs13177623, rs12654195 and rs11168100 had

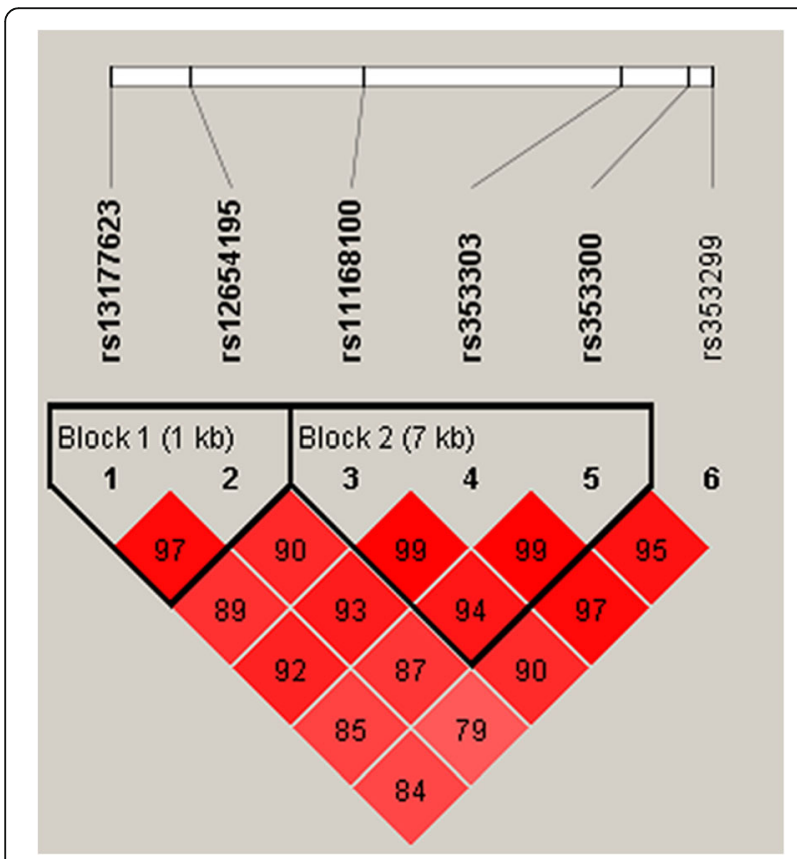

Fig. 1 Linkage disequilibrium (LD) analysis of SNPs in CARMEN. The $L D$ value is determined by $r^{2}>0.8$ analyzed by Haploview software 4.2. The number in the diamonds is the LOD score of $r^{2}$. Standard color schemes indicated different levels of LD. Bright red: $\mathrm{LOD}>2, \mathrm{D}^{\prime}=1$ significantly different levels of cholinesterase, globulin, percentage of neutrophils, and the absolute value of lymphocytes.

CARMEN (also named as MiR143HG), was differentially expressed in cardiac progenitor cells and proliferating cells in cardiovascular pedigree, which was first described by Ouzain et al. [28]. It belongs to the intergenic lncRNA group of ncRNAs and has multiple exon splicing variants. And a previous analysis of promoter specific histone modification and poly (a) signal frequency indicated that the boundaries of two CARMEN transcripts were clear [29]. Chromatin state analysis showed that the expression of MiR143HG isoforms was up-regulated in the process of cardiogenic differentiation between mesoderm and cardiac precursor cell (CPC) [30]. Consistent with this observation, Ounzain et al. found that it was not only expressed in the process of myocardial differentiation, but also in the adult mouse and human heart [31]. MiR143HG knockdown by shRNA or GapmeR can influence the significant reduction of cardiac differentiation markers to impair CPC differentiation [28]. The above research showed that MiR143HG played a role in heart disease, which may affect the occurrence and development of the disease by affecting blood circulation. Our study was the first to show that MiR143HG was related to the risk of necrosis of the femoral head, and the cause of necrosis of the femoral head is the obstruction of microvascular circulation. Although we did not find a link between CARMEN variants and alcohol-induced ONFH risk in people over 45 years old, CARMEN variants were found to be related to a reduced risk of alcohol-induced ONFH in people less than 45 years, which may be related to their relatively smooth blood circulation. Therefore, we speculate that MiR143HG had a certain role in the microvascular circulation of necrosis of the femoral head.

In conclusion, we found that CARMEN variants were associated with alcohol-induced ONFH risk. It proved 
that CARMEN may play a crucial role in the occurrence of ONFH. In spite of some limitations, our results are helpful for the follow-up study of alcohol-induced osteonecrosis of the femoral head. In future, we will use larger samples and rat models of alcoholic osteonecrosis to verify the results.

\section{Conclusion}

In conclusion, the study provides new light on the correlation of CARMEN polymorphisms with alcoholinduced ONFH risk in the Chinese Han population.

\section{Supplementary information}

Supplementary information accompanies this paper at https://doi.org/10. 1186/s12891-020-03553-2.

Additional file $\mathbf{1}$ Supplementary Table $\mathbf{1}$ The primers information of selected SNPS

Additional file $\mathbf{2}$ Supplementary Table $\mathbf{2}$ Association between CARM EN variants and ONFH risk in patients with different clinical stages

Additional file $\mathbf{3}$ Supplementary Table $\mathbf{3}$ The relationship between genotypes of different loci and clinical parameters

Additional file $\mathbf{4}$ Supplementary Table 4 CARMEN haplotypes frequencies associated with ONFH risk

\section{Abbreviations}

SNPs: Single nucleotide polymorphisms; OR: Odds ratio; 95\%Cl: 95\% Confidence interval; C: ARMENCardiac mesoderm enhancer-associated noncoding RNA; LD: Linkage disequilibrium; HWE: Hardy-Weinberg equilibrium; MAF: Minor allele frequency

\section{Acknowledgements}

The authors thank all participants and volunteers of the Zhengzhou Traditional Chinese Hospital of Orthopaedics in this study.

\section{Authors' contributions}

YC G, YJ C and SG G completed the genotyping and performed the draft. SM Z and FZ H participated in the data collation and analysis. XJ Z and JG H collected the samples. ZM Y, JJ Y, DL, XF C and JB S revised the manuscript. YJ C designed the study and co-supervised the work. All the authors have read and approved the final manuscript.

\section{Funding}

This study was supported by TCM Scientific Research Subject of Chinese Medicine Administration Bureau of Henan (2018ZY3011). The funding provided by the funders ensured the smooth implementation of the research.

\section{Availability of data and materials}

The datasets used and/or analysed during the current study are available from the corresponding author on reasonable request.

\section{Ethics approval and consent to participate}

The protocol has been approved by the Ethics Committee of the Zhengzhou Traditional Chinese Hospital of Orthopaedics (20180802). All participants have signed informed consent prior to participating in the study. Meanwhile, our study strictly conformed to the principles of the Declaration of Helsinki.

\section{Consent for publication}

Not Applicable.

\section{Competing interests}

The authors declare that they have no competing interests.
Received: 3 January 2020 Accepted: 31 July 2020

Published online: 15 August 2020

\section{References}

1. Mouzas OD, Zibis AH, Bonotis KS, Katsimagklis CD, Hadjigeorgiou GM, Papaliaga MN, Dimitroulias AP, Malizos KN. Psychological distress, personality traits and functional disability in patients with osteonecrosis of the femoral head. J Clin Med Res. 2014;6(5):336-44.

2. Shah KN, Racine J, Jones LC, Aaron RK. Pathophysiology and risk factors for osteonecrosis. Curr Rev Musculoskelet Med. 2015;8(3):201-9.

3. Zalavras CG, Lieberman JR. Osteonecrosis of the femoral head: evaluation and treatment. J Am Acad Orthop Surg. 2014;22(7):455-64.

4. Adesina OO, Brunson A, Keegan THM, Wun T. Osteonecrosis of the femoral head in sickle cell disease: prevalence, comorbidities and surgical outcomes in California. Blood Adv. 2016;1(16):1287-95.

5. Kubo T, Ueshima K, Saito M, Ishida M, Arai Y, Fujiwara H. Clinical and basic research on steroid-induced osteonecrosis of the femoral head in Japan. $J$ Orthop Sci. 2016;21(4):407-13.

6. Zhao D, Yu M, Hu K, Wang W, Yang L, Wang B, Gao X, Guo Y, Xu Y, Wei Y. Prevalence of nontraumatic osteonecrosis of the femoral head and its associated risk factors in the Chinese population: results from a nationally representative survey. Chin Med J. 2015:128(21):2843-50.

7. Choi HR, Steinberg ME, Cheng EY. Osteonecrosis of the femoral head: diagnosis and classification systems. Curr Rev Musculoskelet Med. 2015;8(3): 210-20.

8. Wang L, Pan H, Zhu ZA. A genetic pedigree analysis to identify gene mutations involved in femoral head necrosis. Mol Med Rep. 2014;10(4): $1835-8$

9. Xue $Y$, Zhao Z, Hong D, Zhang H, Chen H, Fan S. MDR1 gene polymorphisms are associated with glucocorticoid-induced avascular necrosis of the femoral head in a Chinese population. Genet Test Mol Biomarkers. 2014;18(3):196-201.

10. Tsai S, Wu P, Chen C, Chiang C, Huang C, Chen T, Liu C, Chen W. Etiologies and outcome of osteonecrosis of the femoral head: etiology and outcome study in a Taiwan population. Journal of The Chinese Medical Association. 2016;79(1):39-45.

11. Cui L, Zhuang Q, Lin J, Jin J, Zhang K, Cao L, Lin J, Yan S, Guo W, He W. Multicentric epidemiologic study on six thousand three hundred and ninety five cases of femoral head osteonecrosis in China. Int Orthop. 2016:40(2): 267-76.

12. An F, Du J, Wang J, Zhao L, Ma C, Zhao J, Wang J. MMP20 single-nucleotide polymorphisms correlate with susceptibility to alcohol-induced osteonecrosis of the femoral head in Chinese males. Med Sci Monit. 2019; 25:3750-61

13. Liu C, An F, Cao Y, Wang J, Tian Y, Wu H, Wang J. Significant association between RETN genetic polymorphisms and alcohol-induced osteonecrosis of femoral head. Mol Genet Genomic Med. 2019;7(8):e822.

14. Wang Y, Cao Y, Li Y, Guo Y, Wang Q, Yang M, Zhang N, Jin T, Wang J. Genetic association of the ApoB and ApoA1 gene polymorphisms with the risk for alcohol-induced osteonecrosis of femoral head. Int J Clin Exp Pathol. 2015;8(9):11332-9.

15. Wang $Y$, Yang $X$, Shi J, Zhao $Y$, Pan L, Zhou J, Wang G, Wang J. Combination analysis of NOS3, ABCB1 and IL23R polymorphisms with alcohol-induced osteonecrosis of the femoral head risk in Chinese males. Oncotarget. 2017;8(20):33770-8.

16. Chen J, Liu W, Cao Y, Zhang X, Guo Y, Zhu Y, Li J, Du J, Jin T, Wang G, et al. MMP-3 and MMP-8 single-nucleotide polymorphisms are related to alcoholinduced osteonecrosis of the femoral head in Chinese males. Oncotarget. 2017:8(15):25177-88

17. Li Y, Wang Y, Guo Y, Wang Q, Ouyang Y, Cao Y, Jin T, Wang J. OPG and RANKL polymorphisms are associated with alcohol-induced osteonecrosis of the femoral head in the north area of China population in men. Medicine. 2016:95(25):e3981

18. Gibb EA, Brown CJ, Lam WL. The functional role of long non-coding RNA in human carcinomas. Mol Cancer. 2011:10(1):38

19. Su SC, Reiter RJ, Hsiao HY, Chung WH, Yang S. Functional interaction between melatonin signaling and noncoding RNAs. Trends Endocrinol Metab. 2018;29(6):435-45.

20. Klattenhoff CA, Scheuermann JC, Surface LE, Bradley RK, Fields PA Steinhauser ML, Ding H, Butty VL, Torrey L, Haas S. Braveheart, a long 
noncoding RNA required for cardiovascular lineage commitment. Cell. 2013; 152(3):570-83.

21. Ounzain S, Micheletti R, Beckmann T, Schroen B, Alexanian M, Pezzuto I, Crippa S, Nemir M, Sarre A, Johnson R. Genome-wide profiling of the cardiac transcriptome after myocardial infarction identifies novel heartspecific long non-coding RNAs. Eur Heart J. 2014;36(6):353-68.

22. Du C, Shen Z, Zang R, Xie H, Li H, Chen P, Hang B, Xu X, Tang W, Xia Y. Negative feedback circuitry between MIR143HG and RBM24 in Hirschsprung disease. Biochim Biophys Acta. 2016;1862(11):2127-36.

23. Matsuo K, HIROHATA T, SUGIOKA Y, IKEDA M, FUKUDA A. Influence of alcohol intake, cigarette smoking, and occupational status on idiopathic osteonecrosis of the femoral head. Clin Orthop Relat Res. 1988;234:115-23.

24. Totty WG, Murphy WA, Ganz W, Kumar B, Daum WJ, Siegel BA. Magnetic resonance imaging of the normal and ischemic femoral head. Am J Roentgenol. 1984;143(6):1273-80.

25. Du J, Jin T, Cao Y, Chen J, Guo Y, Sun M, Li J, Zhang X, Wang G, Wang J. Association between genetic polymorphisms of MMP8 and the risk of steroid-induced osteonecrosis of the femoral head in the population of northern China. Medicine. 2016;95(37):e4794.

26. Purcell S, Neale BM, Toddbrown K, Thomas L, Ferreira MAR, Bender D, Maller J, Sklar P, De Bakker PIW, Daly MJ. PLINK: a tool set for whole-genome association and population-based linkage analyses. Am J Hum Genet. 2007; 81(3):559-75.

27. Yu Y, Xie Z, Wang J, Chen C, Du S, Chen P, Li B, Jin T, Zhao H. Singlenucleotide polymorphisms of MMP2 in MMP/TIMP pathways associated with the risk of alcohol-induced osteonecrosis of the femoral head in Chinese males: a case-control study. Medicine (Baltimore). 2016;95(49). e5407.

28. Ounzain S, Micheletti R, Arnan C, Plaisance I, Cecchi D, Schroen B, Reverter F, Alexanian M, Gonzales C, Ng SY, et al. CARMEN, a human super enhancerassociated long noncoding RNA controlling cardiac specification, differentiation and homeostasis. J Mol Cell Cardiol. 2015;89(Pt A):98-112.

29. Derrien T, Johnson R, Bussotti G, Tanzer A, Djebali S, Tilgner H, Guernec G, Martin D, Merkel A, Knowles DG. The GENCODE v7 catalog of human long noncoding RNAs: analysis of their gene structure, evolution, and expression. Genome Res. 2012;22(9):1775-89.

30. Boucher JM, Peterson SM, Urs S, Zhang C, Liaw L. The miR-143/145 cluster is a novel transcriptional target of Jagged-1/notch signaling in vascular smooth muscle cells. J Biol Chem. 2011;286(32):28312-21.

31. Ounzain S, Micheletti R, Beckmann T, Schroen B, Alexanian M, Pezzuto I, Crippa S, Nemir M, Sarre A, Johnson R, et al. Genome-wide profiling of the cardiac transcriptome after myocardial infarction identifies novel heartspecific long non-coding RNAs. Eur Heart J. 2015;36(6):353-368a.

\section{Publisher's Note}

Springer Nature remains neutral with regard to jurisdictional claims in published maps and institutional affiliations.

Ready to submit your research? Choose BMC and benefit from:

- fast, convenient online submission

- thorough peer review by experienced researchers in your field

- rapid publication on acceptance

- support for research data, including large and complex data types

- gold Open Access which fosters wider collaboration and increased citations

- maximum visibility for your research: over $100 \mathrm{M}$ website views per year

At $\mathrm{BMC}$, research is always in progress.

Learn more biomedcentral.com/submissions 